\title{
PERSPECTIVES ON GENE THERAPY IN THE TREATMENT OF OCULAR INFLAMMATION
}

\author{
ROBERT B. NUSSENBLATT and KARL CSAKY \\ Bethesda, Maryland
}

\begin{abstract}
SUMMARY
Gene therapy may become a powerful therapeutic strategy. However, the application of this method in the treatment of ocular disease presents us with interesting and unique questions. Gene therapy for ocular inflammatory disease has the potential for both therapeutic interventions and a method for studying mechanisms of disease. An evolving philosophy on this subject would support the use of somatic gene therapy for ocular inflammatory disease, even if not life threatening. Major technical questions remain, including the use of the appropriate vector, the best methodology for the stable insertion into the genome, and the duration and intensity of expression of the transgene. Various transgenes encoding a wide variety of proteins can be envisaged for the insertion of genes. The study of gyrate atrophy, an hereditary ocular disorder and an excellent candidate for gene therapy, has given us enormous information in the development of practical therapeutic strategies, as have in vitro studies of gene insertion. Future concerns will need to concentrate on the use of better methods for gene insertion and homologous recombination techniques for the development of animal models and later as a strategy for gene therapy. The use of gene therapy as a drug delivery system must also be considered. In addition, the elucidation of the various events controlling transcription for the expression of transgenes in various resident ocular cells is necessary.
\end{abstract}

Strategies for gene insertion have become a recurring theme in essentially every branch of medicine. The potential clinical application for this therapeutic approach is enormous. However, today's technology places serious constraints on what can be accomplished. Added to the technological limitations are the serious philosophical and societal considerations that will need to be discussed before gene therapy becomes widely used. This review will discuss how

Correspondence to: R. B. Nussenblatt, MD, National Eye Institute, 10 Center Drive, MSC-1858, Building 10 Room 10N202, Bethesda, MD 20892, USA. gene therapy could be applied to the treatment of ocular inflammatory disease. It will attempt to review what is possible today, using in particular the experience our laboratory has gained in developing a strategy for the treatment of other ocular disorders, mostly hereditary diseases, such as gyrate atrophy. It will then touch upon many of the points at which intervention with this strategy might be possible in the treatment of various ocular conditions that are immune-mediated.

The application of gene therapy to the treatment of disease has raised many cultural and religious concerns. Many position papers and meetings have dealt with this question. Some points have been repeatedly supported by all those who have written or debated the subject. The use of somatic gene therapy has been almost universally agreed on as an acceptable approach, with countries and religious groups (at least in the West) issuing reports supporting this notion. In contrast, the insertion of genes into the germ line has not been sanctioned for human use, though it has been done in mice. For us in ophthalmology, a second issue would be the use of this technique in the treatment of non-life-threatening disorders. The use of gene therapy in the case of a child with a severe immunodeficiency or an inoperable cancer can easily be supported. However, ethicists who have evaluated this question generally conclude that enough work has been done to indicate that the relative safety of today's gene therapy technology warrants its use in non-life-threatening conditions such as blindness.

Using today's standards, several criteria would need to be followed in order to justify the use of gene therapy in man. Four major goals need to be met: (1) A relatively clear idea of the disease's natural history in man is needed. For many ocular inflammatory diseases we know that untreated disease will lead to severe visual impairment. (2) The gene or genes responsible for the disorder needs to be identified. For ocular inflammatory disease we are aware of 
several major mechanisms in the immune system that are believed to play important roles in either the inflammatory process or the ensuing destruction. (3) The existence or the development of in vitro and animal models to test the underlying logic of a proposed gene insertion is required. We are fortunate to have several animal models for ocular inflammatory disease. Some may be developed through the use of homologous recombination, in order to test specific questions, as has been done recently by collaborators of ours. ${ }^{1}$ (4) Finally, there is the gene insertion into patients. This decision is dependent on many factors, including the severity of the disease, the availability, efficacy and side-effects of present therapy, the availability of vectors that can safely insert the gene in question (risk/benefit ratio), and the cost. For many of the disorders we will mention in this review, the dysfunction appears to be localised to the posterior segment, raising important questions about gene delivery and safety.

\section{TODAY'S STRATEGIES}

Technology is constantly changing, and we feel that it is reasonable to be cautiously optimistic concerning rapid changes in our approach to gene therapy. However, the ideal disorder for consideration would be a disease that is known to be caused by only one gene defect, and the gene product being measurable (Table I). Additionally, since gene therapy today is aimed at replacing one altered gene, it would make sense that the ideal candidate disorder is one in which the gene defect leads to no production of a product. This is ideal since it is possible that in disorders where there is a mutated gene, the altered product is itself toxic, so that transgenic insertion producing a normal product may not be helpful. We would ideally wish to consider a disease that has a known natural history so that if an intervention is contemplated its relative efficacy can be meaningfully evaluated. Finally, the disorder should have either no effective therapy or that therapy (or therapies) should have a poor outcome or side effects of some consequence. We must at this time consider only somatic gene therapy. Finally, the existence of animal models facilitates matters enor-

Table I. Characteristics of an ideal candidate for gene therapy today

Gene causing disease has been isolated and sequenced

Disease process can be recognised early

No toxic product is produced by a mutated gene or genes

Existing treatment is costly, partially effective, but does show some effectiveness

Gene has a simple regulation

Natural history of the disease is known, so that any intervention can be evaluated

Treatment strategy lends itself particularly to an ex vivo approach, but in vivo can be considered

Ability to follow a marker for gene activity once it has been inserted mously, since they help to elucidate mechanisms and can be a testing ground for therapeutic interventions.

Viral vectors are today's choice for gene insertion. Though efficient in their insertion capacity, they possess numerous negative characteristics. Initial work performed in humans used a modified retrovirus vector for gene insertion. This work was performed ex vivo. The advantage of the retrovirus is that it has the longest track record in humans, and the insertion appears to be quite stable. Essentially only one insertion of the gene in question occurs and therefore the production of the protein desired may be low. A major drawback is that its insertion is dependent on cell division. Since many of the cells in the eye that we may target for in vivo gene therapy are non-dividing end-stage cells, this method will not be practical for many indications. The viral vector that is receiving the most attention at the moment is that of adenovirus. As this is capable of inserting numerous copies of the same gene into a cell, the amount of protein production can theoretically be very high. Additionally gene insertion is not dependent on cell multiplication. To date most studies have used an E1-deficient recombinant adenoviral vector. The E1A and E1B genes are thought to control virus replication and the expression of many early and late genes. ${ }^{2}$ While expression may be high in a variety of cellular targets, it is usually for a limited period of time. Since transgenes are not integrated into the cell genome, cell division theoretically could 'dilute' the effect. However, a more important drawback has been the immune response directed against the proteins of the adenovirus itself, which appears to limit the long-term usefulness of the E1-deficient vector. It has been noted that the ablation of the E2A gene in recombinant adenoviruses improves transgene persistence and decreases the inflammatory response, at least in mice. ${ }^{3}$ In passing it should be mentioned that a third virus, the adenoassociated virus, has received some attention because of the lack of an apparent immune response directed against it. ${ }^{4,5}$ However, technical problems continue and its potential use seems to be uncertain.

Bearing in mind the considerations outlined above, we have identified what we believe is the ideal initial ocular candidate for somatic gene therapy. Unlike others, we do not feel that disorders such as retinitis pigmentosa would be best approached by today's methodology. In retinitis pigmentosa an abnormal, harmful mutant is produced, making it unclear whether the insertion of the normal gene will have any benefit. Problems still need to be solved as to how best to insert the transgene into the retina. Gyrate atrophy of the retina and choroid is an autosomal recessive chorioretinal degeneration caused by a deficiency of the nuclear-encoded mitochondrial matrix enzyme, ornithine-D-amino- 
transferase (OAT). Scalloped peripheral chorioretinal lesions appear early in life, and in the disease the individual is night blind with a marked diminution of the electroretinogram. Treatment with a lowarginine diet has been shown to stop or slow the course of the disease. ${ }^{6}$ However, the diet is very difficult to follow. Using homologous recombination techniques, an OAT-deficient mouse model of gyrate atrophy has been shown to develop retinal pigment epithelium (RPE) damage and progressive retinal degeneration. ${ }^{1}$

We have adapted a strategy that will use the skin of gyrate atrophy patients as the target for gene therapy with $\mathrm{OAT}^{7}$ with continuing experiments looking at the possibility of direct gene insertion into the RPE. Skin as a target for gene therapy has certain advantages, including its availability, the possibility of performing the transductions ex vivo, the stability of the transductions, and the ability to follow these altered cells carefully. Much information has been gained from initial ex vivo experiments. Firstly, with the successful ex vivo transduction of keratinocytes from patients with gyrate atrophy we have demonstrated the feasibility of this approach, i.e. the use of skin as a possible site for an OAT enzyme or source. These studies will continue soon with attempts to place transduced keratinocytes back in the original gyrate atrophy donors.

We have also gained much information about the potential pitfalls of gene therapy for at least one potential site: the RPE. Primary cultures of human RPE have been established and ultimately transduced using an adenovirus vector containing the human OAT cDNA under the control of the human cytomegalovirus promoter (pOATAd.CMV.1). This vector, deficient in the E1A and E1B regions, can be isolated in large quantities with titres in excess of $10^{12}$ particles/ml. Transduction of the RPE with pOATAd.CMV1 was very efficient, with these cells increasing their baseline OAT levels 350 -fold higher than the baseline of $65 \mathrm{nmol} / \mathrm{mg}$ protein per hour (to $24000 \mathrm{nmol} / \mathrm{mg}$ protein per hour). ${ }^{8}$ However, evidence of mitochondrial toxicity, at the highest OAT levels, was noted, as evidenced by a significant drop in succinate dehydrogenase activity. Therefore, on the basis of these studies we have been able to maintain an extremely high level of expression (250fold higher than baseline) without inducing obvious toxicity of the cells.

These studies have revealed important points that need to be considered when gene insertion is contemplated. There is the concern of toxicity induced by the vector itself; overproduction of the desired protein may lead to mitochondrial toxicity. Secondly, the 'flux' of the substrate that is desired must be considered. Increasing or introducing a particular enzyme may not necessarily result in an equivalent increase in substrate metabolism, since other factors may impede substrate production elsewhere. There is also the possibility that introducing a new protein may lead to an immune response.

\section{STRATEGIES FOR OCULAR INFLAMMATION}

One can envisage gene therapy for the whole gamut of ocular inflammation, that is for autoimmune disease, infection and tumour. Most of our comments will be limited to disease that affects the posterior segment of the eye, but it is noteworthy to mention therapy to the cornea. Diseases of the cornea are particularly amenable to gene therapy since vectors could readily be placed onto the corneal epithelium. Treatment for not only hereditary disease but also inflammatory disorders such as herpes keratitis, autoimmune corneal melts and rejection phenomena due to corneal transplantation would be readily approachable. Specific corneal promoters are being identified (J. Piatigorsky, personal communication) which will be used shortly in conjunction with vectors for gene transduction.

As regards the posterior segment, the development of an ideal vector system is still required. Some evidence suggests that, because of the special immune characteristics of the eye, no inflammatory reaction would occur with the placement of adenovectors. ${ }^{9}$ However, experiments testing this possibility need to be completed. Since long-term expression with adenovirus is not possible, reinjection of the adenovirus vector is required for extended therapeutic approaches. The initial placement of virus may cause immune priming, with the second therapy potentially inducing an anamnestic immune response.

Taking into account that the ideal method for gene insertion has yet to be found, several approaches for the treatment of autoimmune disease involving the posterior segment can be considered.

1. Cytokine Insertion. This possibility has already begun to be explored. Immunosuppressive cytokines such as transforming growth factor beta-1 (TGF- $\beta 1$ ) and interleukin-10 (IL-10) have been used in ex vivo experiments. An E1-deleted adenovirus vector, which can transduce a constitutively active form of TGF- $\beta 1$, has been constructed. Significant biological quantities of the cytokine can be produced by human RPE cells. It would be quite possible to transduce organ-resident cells such as the RPE or cells in the ciliary body in order to either downregulate an immune response or to recreate an immunosuppressive environment such as ACAID.

2. Apoptosis. Programmed cell death can be seen as both being of aid and also being very harmful during and after an intraocular inflammatory episode. A Fas(CD95)-Fas ligand interaction leading to apoptosis has been shown to occur in the eye. ${ }^{10}$ 
Certainly attempts to induce apoptosis in invading inflammatory cells could be a possibility, but technically this may be difficult to do. However, an inflammatory episode will lead to photoreceptors undergoing apoptosis. The insertion of factors to prevent the apoptotic process could be a helpful post-inflammatory treatment to save photoreceptors.

3. Regenerative Factors. In vitro experiments have identified pigment epithelial derived growth factor (PEDF) as a potential neurite survival factor. ${ }^{11}$ In addition, other growth factors such as basic fibroblast growth factor (bFGF) have been shown to rescue photoreceptors in an animal model of retinal degeneration. $^{12}$ Therefore, the use of adenovirus vectors expressing these factors might find a role in photoreceptor regeneration.

4. Insertion of Drugs. The insertion of non-human genes into human cells is no longer impossible. ${ }^{13}$ However, the insertion of such genes raises many questions. The appropriate cellular machinery needs to be present so that production of an active molecule can take place. Also, it is not inconceivable that an immune response could be directed against that new, foreign protein. However, it is possible to consider inserting immunosuppressives into cells, particularly those that do not require any posttranslational modifications.

5. Transcription Factors. This will certainly be a very important area of study in the coming years. The factors that are responsible for the activation or inhibition of expression will be an area that is ideal for control of the intraocular immune response. Initial studies have begun to delineate this area. ${ }^{14}$ Interactions between the immune system and resident ocular cells have begun to be elucidated. The role of interferon on ocular resident cells has been evaluated both in a transgenic model ${ }^{15}$ and in models of experimental uveitis. Egwuagu and associates ${ }^{16,17}$ have shown that the effect of interferon-gamma on gene regulation in ocular tissues appears to be mediated by transcription factors induced by this cytokine. Interferon regulatory factor (IRF) 1 and 2 as well as interferon consensus sequence binding protein (ICSCB) (positive and negative regulatory factors) are activated in the eye in the presence of interferon-gamma. Egwuagu et al. have further shown that the negative regulatory factors ICSCB and IRF-2 are activated in RPE cells but lens epithelial cells appear to produce only ICSCB. Thus, differences in the transcription factor response on the part of ocular inflammatory cells have begun to be discerned, and are being studied in several contexts, including the ability of DNA viruses to establish latent and active infections in RPE cells. This area will be ripe for the exploration of 'inducible' genes, which could regulate elements that respond to changes in the environment, such as to an antibiotic or a hormone. ${ }^{13}$

Important regulators of the expression of various immune-related proteins have been identified, though their exact role in the eye still needs to be determined. One factor that will certainly be the focus of such an intervention will be activator protein-1 (AP-1). This transcription factor binds into a complex which increases IL-2 expression. A second factor, nuclear factor-kB, will influence genes that are activated by extracellular signals, including inducible nitric oxide, cyclo-oxygenase 2 , IL-8, and adhesion molecules. Both factors, AP-1 and nuclear factor-kB, can block activated glucocorticoid receptors, thereby reducing the effect of steroid on an immune response.

\section{INFECTION}

One can easily envisage the use of gene therapy that can be applied in cases of severe infection. In cases of endophthalmitis, once the vitrectomy has been performed, vectors containing genes that either prevent apoptosis of retinal elements or induce neuroregenerative responses could be introduced:

\section{TUMOUR}

Many gene insertion strategies are being considered for the treatment of tumours. One of the most attractive strategies involves those genes which would induce apoptosis in tumour cells. This method often requires interaction with other cells expressing the complementary apoptotic antigen. However, another approach we have already begun investigating is the insertion of transgenes that render tumours treatable with antibiotics. One such approach is the insertion of the thymidine kinase gene, which renders the tumour susceptible to treatment with ganciclovir. We are exploring the use of this method in melanoma cells. The development of an animal model for ocular melanoma has helped us enormously in developing this methodology.

In summary, we have just begun the long road that will lead to the treatment of patients with ocular disease using the methods of gene insertion. We have tried here to demonstrate the potential problems that need to be confronted before this method can be applied in humans. However, it is clear that while the ideal way to introduce transgenes into ocular tissue needs still to be defined, the potential is very real that this methodology will be used to treat patients with ocular inflammatory disease.

We take this opportunity to thank our colleagues, whose hard. work has permitted these ideas to be put to paper: Drs Daniel Sullivan and Muriel Kaiser, National Eye Institute; Dr Michael Blaese, National Center for Human Genome Research; Dr Lorne Taichman, SUNY-Stonybrook; and Dr David Valle, Johns Hopkins University School of Medicine. 
Key words: Ocular disease, Gene therapy, Uveitis, Autoimmune disease.

\section{REFERENCES}

1. Wang T, Milam AH, Steel G, Valle D. A mouse model of gyrate atrophy of the choroid and retina. J Clin Invest 1996;97:2753-62.

2. Kozarsky KF, Wilson JM. Gene therapy: adenovirus vectors. Curr Opin Genet Dev 1993;3:499-503.

3. Engelhardt JF, Ye X, Doranz B, Wilson JM. Ablation of E2A in recombinant adenoviruses improves transgene persistence and decreases inflammatory response in mouse liver. Proc Natl Acad Sci USA 1994;91: 6196-200.

4. Morgan RA, Anderson WF. Human gene therapy. Annu Rev Biochem 1993;62:191-217.

5. Cornetta K, Morgan RA, Anderson WF. Safety issues related to retroviral-mediated gene transfer in humans. Hum Gene Ther 1991;2:5-14.

6. Kaiser-Kupfer M, Caruso R, Valle D. Gyrate atrophy of the choroid and retina: chronic reduction of ornithine slows retinal degeneration. Arch Ophthalmol 1991;109:1539-48.

7. Vogel JC. Keratinocyte gene therapy. Arch Dermatol 1993;129:1478-83.

8. Sullivan DM, Chung DC, Anglade E, Nussenblatt RB, Csaky KG. Adenovirus-mediated gene transfer of ornithine aminotransferase in cultured human retinal pigment epithelium. Invest Ophthalmol Vis Sci 1996;37:766-74.

9. Bennett J, Wilson J, Sun D, Forbes B, Maguire A.
Adenovirus vector-mediated in vivo gene transfer into adult murine retina. Invest Ophthalmol Vis Sci 1994;35:2535-42.

10. Griffith TS, Brunner T, Fletcher SM, Green DR, Ferguson TA. Fas ligand-induced apoptosis as a mechanism of immune privilege. Science 1995;270: 1189-92.

11. Tombran-Tink J, Chader GG, Johnson LV. PEDF: a pigment epithelium-derived factor with potent neuronal differentiative activity. Exp Eye Res 1991;53:411-4.

12. Unoki K, LaVail MM. Protection of the rat retina from ischemic injury by brain-derived neurotrophic factor, ciliary neurotrophic factor, and basic fibroblast growth factor. Invest Ophthalmol Vis Sci 1994;35:907-15.

13. Blau HM, Springer ML. Gene therapy: a novel form of drug therapy. N Engl J Med 1995;333:1204-7.

14. Papavassiliou AG. Transcription factors. N Engl J Med 1995;332:45-7.

15. Egwuagu CE, Sztein J, Reid W, et al. Gamma interferon gene expression in the lens of transgenic mice directed by the alpha-A-crystallin gene promoter. Invest Ophthalmol Vis Sci 1992;33:846.

16. Egwuagu CE, Sztein J, Chan CC, et al. Gamma interferon expression disrupts lens and retinal differentiation in transgenic mice. Dev Biol 1994;166: 557-68.

17. Egwuagu CE, Mahdi R, Smith JA, Chan CC, Chepelinsky AB. Gamma interferon-expressing transgenic rats: a useful model of anterior and posterior uveitis. Invest Ophthalmol Vis Sci 1996;37(Suppl): 4229. 